\title{
Effects of gamma-ray radiation on channel current of the uniaxial strained $\mathrm{Si}$ nano-scale NMOSFET
}

\author{
Minru Hao ${ }^{\text {a) }}$, Huiyong Hu, Chenguang Liao, and Bin Wang \\ School of Microelectronics, Xidian University, \\ 2 South Taibai Road, Xi'an 710071, P. R. China \\ a)haominru@163.com
}

\begin{abstract}
An analytical model of channel current for the uniaxial strained Si nanometer NMOSFET has been developed with the degradation due to total dose irradiation taken into consideration. Based on this model, the numerical simulation has been carried out by Matlab, and the influence of the total dose on channel current was simulated. Furthermore, to evaluate the validity of the model, the simulation results were compared with experimental data, and good agreements were confirmed. Thus, the proposed model provides good reference for research on irradiation reliability of uniaxial strained Si nanometer NMOSFET.
\end{abstract}

Keywords: uniaxial strained Si, nano NMOSFET, total dose, channel current

Classification: Electron devices, circuits and modules

\section{References}

[1] M. Ray, et al.: "Photoluminescence from oxidized macroporous silicon: Nanoripples and strained silicon nanostructures," IEEE Trans. Device Mater. Rel. 13 (2013) 87 (DOI: 10.1109/TDMR.2012.2214781).

[2] J.-S. Goo, et al.: "Scalability of strained-Si nMOSFETs down to $25 \mathrm{~nm}$ gate length," IEEE Electron Device Lett. 24 (2003) 351 (DOI: 10.1109/LED.2003. 812563).

[3] I. Sanchez Esqueda, et al:: "Compact modeling of total ionizing dose and aging effects in MOS technologies," IEEE Trans. Nucl. Sci. 62 (2015) 1501 (DOI: 10.1109/TNS.2015.2414426).

[4] E. Garcia-Moreno, et al.: "Temperature compensated floating gate MOS radiation sensor with current output," IEEE Trans. Nucl. Sci. 60 (2013) 4026 (DOI: 10.1109/TNS.2013.2277605).

[5] J. R. Schwank, et al:: "Radiation effects in MOS oxides," IEEE Trans. Nucl. Sci. 55 (2008) 1833 (DOI: 10.1109/TNS.2008.2001040).

[6] C. C. M. Bordallo, et al:: "Influence of X-ray radiation on standard and uniaxial strained triple-gate SOI FinFETs," Proc. RADECS (2013) 1 (DOI: 10. 1109/RADECS.2013.6937356).

[7] M. Hao, et al.: "Total ionizing dose radiation effect on the threshold voltage for the uniaxial strained Si Nano NMOSFET," IEICE Electron. Express 14 (2017) 20170411 (DOI: 10.1587/elex.14.20170411).

[8] J.-T. Qu, et al.: "Study of threshold voltage and I-V characteristic for small- 
scaled strained Si/SiGe PMOSFET,” J. Univ. Electron. Sci. Technol. China 41 (2012) 311 (DOI: 10.3969/j.issn.1001-0548.2012.02.026).

[9] R. F. Pierret: Fundamentals of Semiconductor Device, translated by R. Huang, et al. (Publishing House of Electronics Industry, Beijing) (2010) 275.

[10] J. B. Roldan, et al.: "Modeling effects of electron-velocity overshoot in a MOSFET,” IEEE Trans. Electron Devices 44 (1997) 841 (DOI: 10.1109/16. 568047).

\section{Introduction}

Strained technology has been widely used to improve the performance of Si CMOS devices, and strained-Si technologies have caught much attention as device size is scaling down [1,2], especially in the applications of strained IC under the total dose irradiation. Hence, researches on the irradiated characteristics and the radiation hardening technique are of great significance [3, 4]. Channel current characteristic is an important foundation for the analysis of DC, transient, AC small signal, noise of the uniaxial strained Si nanometer NMOSFET device and circuit. A number of papers reported about current of the MOS device under the radiation effect $[5,6]$, which were based on experiment but lack of mature theory. Moreover, the channel current model of the uniaxial strained Si nanometer NMOSFET has rarely been reported under the total dose irradiation. In this paper, the channel current model of the uniaxial strained Si nanometer NMOSFET were proposed due to the total dose radiation.

\section{Channel current model}

The schematic structure of uniaxial strained Si NMOSFET is shown in Fig. 1.

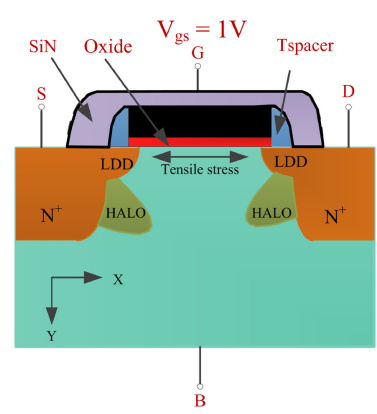

Fig. 1. Structural diagram of uniaxial strained $\mathrm{Si}$ nanometer NMOSFET device.

The threshold voltage model of the uniaxial strained Si nanometer NMOSFET devices after considering the quantum effect and total dose radiation effect is calculated by [7]:

$$
\begin{aligned}
V_{t h, s s i}= & V_{F B, s s i}-V_{D I B L} \\
& +\frac{\psi_{t h, s s i}+\left[1-2 \frac{\sinh \left(\frac{L}{2 l}\right)}{\sinh \left(\frac{L}{l}\right)}\right] \frac{q N_{a}}{\varepsilon_{s i}} l^{2}-\frac{\sinh \left(\frac{L}{2 l}\right)}{\sinh \left(\frac{L}{l}\right)}\left(2 \psi_{b i, s s i}+V_{d s}\right)}{\left[1-2 \frac{\sinh \left(\frac{L}{2 l}\right)}{\sinh \left(\frac{L}{l}\right)}\right]\left[1-\frac{6}{W_{d}^{2}} l^{2}\right]}
\end{aligned}
$$


where $\mathrm{L}, 1$ and $\mathrm{W}_{\mathrm{d}}$ are channel length, characteristics scaling length and the maximum width of the depletion-layer, respectively. $\mathrm{V}_{\mathrm{FB}, \mathrm{ssi}}, \mathrm{V}_{\mathrm{DIBL}}, \psi_{\mathrm{th}, \mathrm{si}}, \psi_{\mathrm{th}, \mathrm{ssi}}$, $\mathrm{N}_{\mathrm{a}}, \mathrm{V}_{\mathrm{ds}}$ and $\varepsilon_{\mathrm{si}}$ are flat band voltage, drain-induced barrier lowering, threshold surface potential of relaxation and strained silicon, doping concentration in the channel, drain voltage and dielectric constant of silicon, respectively. $\mathrm{V}_{\mathrm{FB} \text {,ssi }}$ can be modified due to total dose radiation effect, which is rewritten as:

$$
V_{F B, s s i}=\left(\frac{\phi_{M}-\phi_{s s i}}{q}\right)-\frac{\left(Q_{o x}+q N_{o t}-q N_{i t}\right)}{C_{o x}}
$$

where $\phi_{\mathrm{M}}$ and $\phi_{\mathrm{ssi}}$ are the work function of metal gate and the substrate of strained $\mathrm{Si}$, respectively. $\mathrm{Q}_{\mathrm{ox}}, \mathrm{C}_{\mathrm{ox}}, \mathrm{N}_{\mathrm{ot}}$ and $\mathrm{N}_{\mathrm{it}}$ are the initial density of surface charge and capacitance in the oxide layer, oxide-trapped charge and interface-trap charge, respectively.

Because the channel length is the same magnitude as or less than mean free path of the channel carrier, which are not subject to scattering for the small size device. Therefore, the formation speed is too fast, and the current caused by the velocity overshoot effect can't be ignored. Therefore, the equation of channel current for uniaxial strained $\mathrm{Si}$ nanometer NMOSFET can be obtained by $\mathrm{I}_{\mathrm{ds}}=\left(\mathrm{Wt}_{\mathrm{ssi}}\right) \mathrm{J}$, which is expressed as:

$$
I_{d s}=W t_{s s i} J=W t_{s s i}\left[q u_{n, s s i} n(x) E(x)+q u_{n, s s i} n(x) \delta(E) \frac{d E(x)}{d x}\right]
$$

where $\delta(\mathrm{E}) \approx 2 / 3 \mathrm{v}_{\mathrm{sat}} \tau_{\mathrm{w}}$ is energy relaxation length. $\mathrm{v}_{\mathrm{sat}}, \mathrm{J}, \mathrm{W}, \mathrm{u}_{\mathrm{n}, \mathrm{ssi}}, \mathrm{n}(\mathrm{x})$ and $\mathrm{t}_{\mathrm{ssi}}$ are saturated velocity, current density and gate width, electron mobility, concentration and thickness of strained silicon, respectively. $\mathrm{E}(\mathrm{x})$ and $\tau_{\mathrm{w}}$ are electric field and energy relaxation time, which varies with the magnitude of stress [8]. The electrons are confined to the surface of the quantum well channel, and its concentration can be obtained as:

$$
n(x)=C_{o x}\left(V_{g s}-V_{t h, s s i}-V(x)\right) / q t_{s s i}
$$

where $\mathrm{V}(\mathrm{x})$ is the voltage drop along the channel direction. $\mathrm{V}_{\mathrm{gs}}$ is gate voltage. For the strong electronic field, $u_{s s i}$ is expressed as [9]:

$$
u_{s s i}=u_{0, s s i}\left(1+\frac{u_{0, s s i}}{2 v_{s a t}} E(x)\right)^{-1}
$$

where $\mathrm{u}_{0, \text { ssi }}$ is the low field electron mobility.

The influence of $\gamma$-ray total dose radiation effect on mobility can be obtained by [10]:

$$
\frac{u_{n, s s i}}{u_{s s i}}=\frac{1}{1+a_{i t} N_{i t}}=1 /\left[1+a_{i t} N_{s i-H} \times\left(1-e^{-\frac{1}{2} \sigma_{D H} \sigma_{p t} N_{D H} K_{g} f_{y} t_{0 x}^{2} D^{\prime} t}\right)\right]
$$

where the total dose $D=D^{\prime} t$.t, $\mathrm{K}_{\mathrm{g}}$ and $\alpha_{\mathrm{it}}$ are irradiation time, the number of electron-hole pairs (EHP) generated per unit dose and an empirical constant related to interface state density, respectively. $\mathrm{f}_{\mathrm{y}}, \sigma_{\mathrm{pt}}$ and $\mathrm{N}_{\mathrm{DH}}$ are the hole yield as a function of oxide electric field, the hole capture cross section and the trap concentration of hydrogen, respectively. $\sigma_{\mathrm{DH}}, \mathrm{N}_{\mathrm{Si}-\mathrm{H}}$ and $\mathrm{t}_{\mathrm{ox}}$ are the trapped cross section of holes with hydrogen traps the silicon dangling bond density of hydrogen passivation and oxide thickness, respectively. When the speed is too fast, $(\mathrm{dE}(\mathrm{x})) / \mathrm{dx}=\theta \mathrm{V}_{\mathrm{ds}} / \mathrm{L}^{2}, \theta$ is related to the technology, and its value is about 0.2 . 
Substituting $\mathrm{E}(\mathrm{x})=(\mathrm{dV}(\mathrm{x})) / \mathrm{dx}$ in Eq. (5), the integral analysis of the Eq. (3) is performed, and the channel current $\mathrm{I}_{\mathrm{ds}}$ (linear region and saturation region) of the uniaxial strained Si nanometer NMOSFET device after considering the velocity overshoot effect of small-scale device and total dose radiation effect can be given by:

$$
\begin{aligned}
& I_{d s}=\frac{W u_{n, s s i} c_{o x}}{L\left(1+\frac{u_{n, s s i} V_{d s}}{2 v_{s a t}}\right)} \times\left(\left(\left(V_{g s}-V_{t h, s s i} V_{d s}-\frac{V_{d s}{ }^{2}}{2}\right) \times\left(1+\frac{\theta \delta(E)}{L}\right)+\frac{\theta^{2} \delta(E)}{12 L} V_{d s}{ }^{2}\right)\right. \\
& I_{d s}=\frac{W u_{n, s s} c_{o x}}{L\left(1+\frac{u_{n, s s i}\left(V_{g s}-V_{t h, s s i}\right)}{2 v_{s a t}}\right)} \times\left(\frac{1}{2}\left(V_{g s}-V_{t h, s s i}\right)^{2}\left(1+\frac{\theta \delta(E)}{L}\right)+\frac{\theta^{2} \delta(E)}{12 L}\left(V_{g s}-V_{t h, s s i}\right)^{2}\right)(8)
\end{aligned}
$$

When the device operates in the sub-threshold region, channel current is mainly dominated by diffusion current. Based on reverse charge of the sub-threshold region, sub-threshold current can be obtained by:

$$
I_{d s}=\frac{W}{L} V_{t} u_{n, s s i}\left[Q_{i}(L)-Q_{i}(0)\right]
$$

where $\mathrm{V}_{\mathrm{t}}$ is the thermal voltage. $\mathrm{Q}_{\mathrm{i}}(0)$ and $\mathrm{Q}_{\mathrm{i}}(\mathrm{L})$ are electron areal density of source and drain in channel, respectively. When the device operates in the subthreshold region, the surface potential from the source to the drain can be approximated as constant. By solving the Poisson equation, the surface potential is given by:

$$
\varphi_{s}=\left(-\frac{\gamma}{2}+\sqrt{\frac{\gamma^{2}}{4}+V_{g s}-V_{t h, s s i}}\right)^{2}
$$

where $\gamma=\frac{\sqrt{2 q_{\varepsilon_{s} N_{a}}}}{C_{o x}}$, electron-sheet-density in channel is obtained by:

$$
Q_{i}(X)=\sqrt{\frac{q \varepsilon_{s i} N_{a}}{2 \varphi_{s}}} V_{t} \exp \left(\frac{V_{g s}-V_{t h, s i}}{n V_{t}}\right) \times \exp \left(-\frac{v(x)}{V_{t}}\right)
$$

where $n=1+\frac{\sqrt{\frac{q_{s i \mathrm{~N}} \mathrm{~N}_{a}}{2 \varphi_{s}}}}{C_{o x}}$, thus sub-threshold current of the uniaxial strained Si nanometer NMOSFET device after considering total dose radiation effect is expressed as:

$$
I_{d s}=u_{n, s s i} \frac{W}{L} \sqrt{\frac{q \varepsilon_{s i} N_{a}}{2 \varphi_{s}}} V_{t}^{2}\left[1-\exp \left(-\frac{V_{d s}}{V_{t}}\right)\right] \times \exp \left(\frac{V_{g s}-V_{t h, s s i}}{n V_{t}}\right)
$$

\section{Experiment}

The device of uniaxial strained Si nanometer NMOSTET were irradiated with ${ }^{60} \mathrm{Co}$ $\gamma$-ray at a dose rate of $29 \mathrm{rad}\left(\mathrm{SiO}_{2}\right) / \mathrm{s}$ at room temperature, with a gate bias of $1 \mathrm{~V}$. Device parameters were measured in the dose range from $0.5 \mathrm{kGy}$ to $2.5 \mathrm{kGy}$, using an HP4155B parametric analyzer. Drain voltage $V_{d s}$ is equal to source voltage $V_{s}$ $\left(\mathrm{V}_{\mathrm{ds}}=\mathrm{V}_{\mathrm{s}}=0\right)$. Measurement bias: $\mathrm{V}_{\mathrm{gs}}=0-1 \mathrm{~V}$, scanning voltage $\mathrm{V}_{\text {step }}=0.05 \mathrm{~V}$, $\mathrm{V}_{\mathrm{ds}}=50 \mathrm{mV}, \mathrm{V}_{\mathrm{s}}=0 \mathrm{~V}$. Based on proposed model, numerical simulation has been carried out by Matlab, and the influence of the total dose on channel current was simulated. Furthermore, stress distribution and electron mobility distribution of simulation are made for uniaxial strained Si nanometer NMOSFET using the 
Sentaurus TCAD. Because of the expansion of the silicon nitride film deposited in the source, drain and gate, the strained in the channel is generated. Fig. 2 is the micrograph and schematic cross-section of uniaxial strained $\mathrm{Si}$ nanometer NMOSTET device. TiAl material is used for the gate electrode. The oxide layer is $\mathrm{SiO}_{2}$ and $\mathrm{HfO}_{2}$, the equivalent oxide thickness being $1 \mathrm{~nm}$. The junction depth of source/drain region is $25 \mathrm{~nm}$ and the gate length is $50 \mathrm{~nm}$.

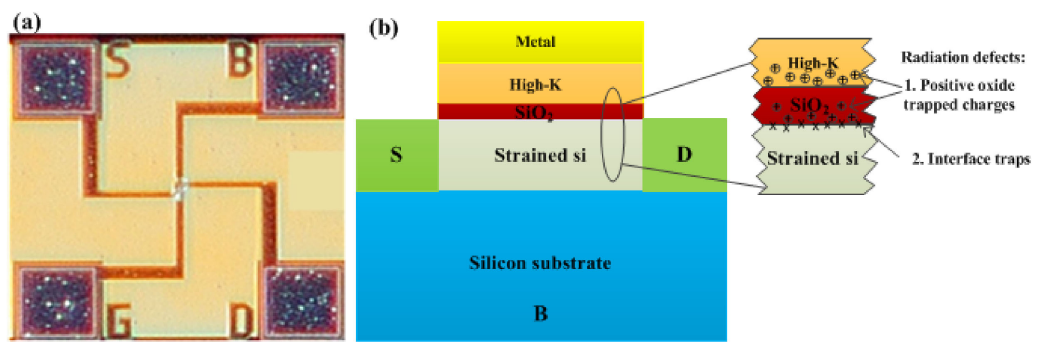

Fig. 2. (a) Micrograph (b) cross-section of uniaxial strained Si Nano NMOSTET

As can be seen from the Table I, the threshold voltage drift of relaxed $\mathrm{Si}$ nanometer NMOSFET is similar to that of uniaxial strained $\mathrm{Si}$ nanometer NMOSFET. It can be inferred that the stress does not change when compared with the case before irradiation.

Table I. The threshold voltage shift of relaxation and uniaxial strained Si nanometer NMOSFET device under the absorbed dose.

\begin{tabular}{l|c|c|c|c|c}
\hline Dose $/ \mathrm{KGy}$ & 0.5 & 1.0 & 1.5 & 2.0 & 2.5 \\
\hline$\Delta \mathrm{V}_{\text {th,si }} / \mathrm{V}$ & -0.0352 & -0.0403 & -0.0410 & -0.0415 & -0.0431 \\
\hline$\Delta \mathrm{V}_{\text {th,ssi }} / \mathrm{V}$ & -0.0354 & -0.0405 & -0.0408 & -0.0417 & -0.0428 \\
\hline
\end{tabular}

\section{Results and discussion}

Fig. 3 shows stress distribution and electron mobility distribution of uniaxial strained Si nanometer NMOSFET device under the pre-irradiation and postirradiation. From Fig. 3(b), as can be seen that electron mobility sharply decreases under the post-irradiation. As is well known, channel current is directly proportional electron mobility. Therefore, as can be inferred that channel current will decrease under the post-irradiation.
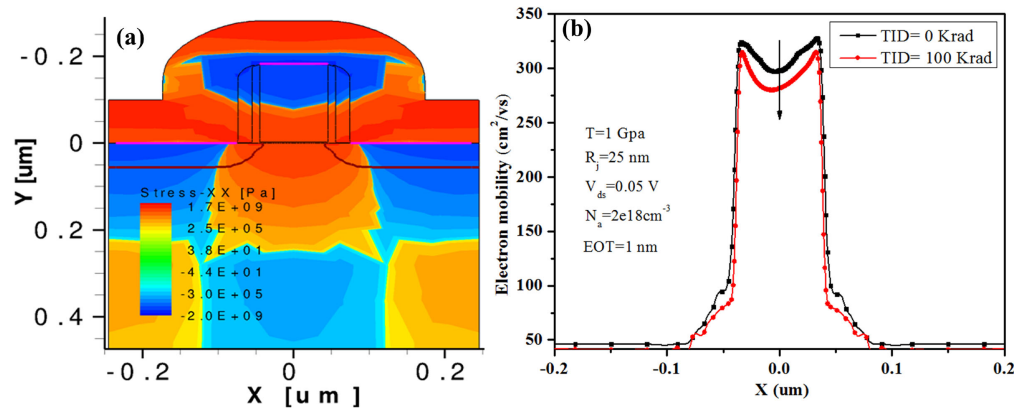
Fig. 4 illustrated the transfer and output characteristics of drain-source current under the different total dose radiation effect. From Fig. 4, as can be seen that channel current decreases with increasing irradiation dose due to electron mobility sharply decreases after irradiation. Moreover, the calculation results from the model are consistent with the experimental data, indicating the validity of our proposed model.
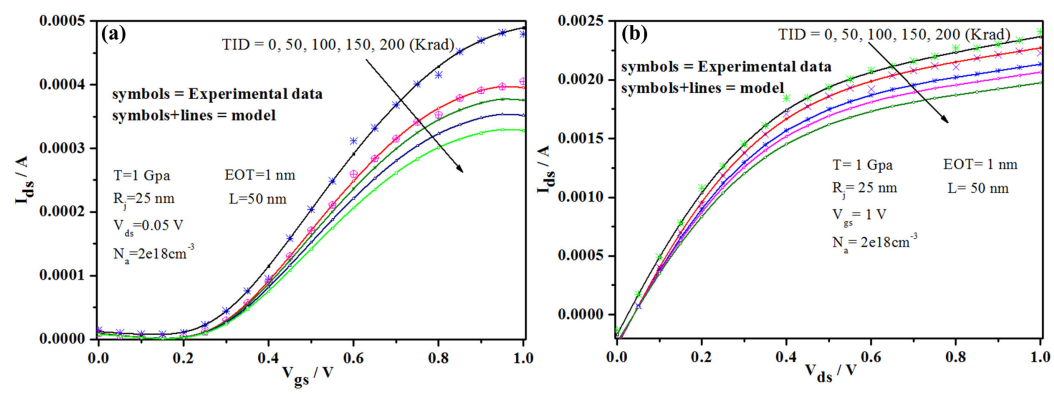

Fig. 4. Transfer and output characteristics under the total dose radiation effect.

Fig. 5 is change of drain current under irradiation. As shown in Fig. 5(a), we can see that Drain-Source current increases with increasing gate voltage, but DrainSource current decreases under the post-irradiation. From Fig. 5(b), sub-threshold current increases under the post-irradiation due to induced oxide-trapped positive charge, which cause the device is more likely to open. Thus, the threshold voltage drifts and the sub-threshold current increases under the post-irradiation.
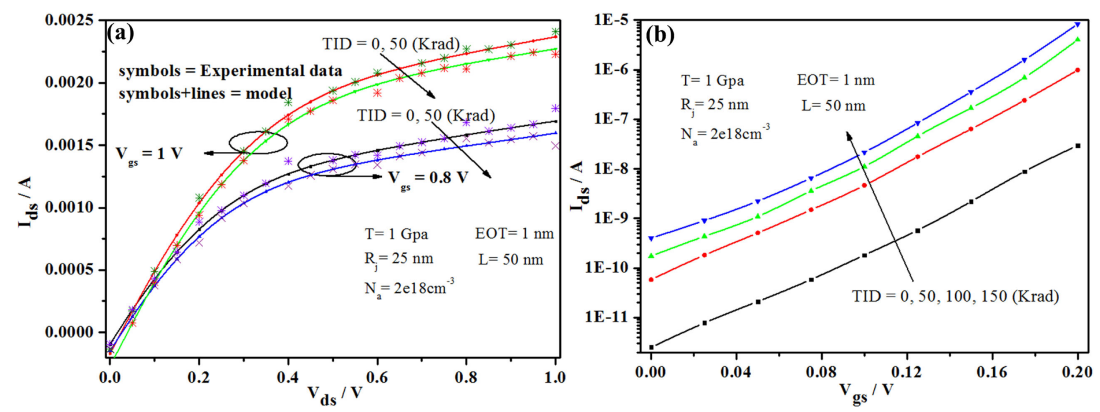

Fig. 5. (a) Results contrast of drain-source current from model and experiment (b) relationship between sub-threshold current and TID.

\section{Conclusion}

Based on the different working regions of the device, the channel current model of the uniaxial strained Si nanometer NMOSFET was established in terms of the basic equation of drift-diffusion, and numerical simulations are carried out by Matlab. The results from the model are compared with the experimental data and they are found to be in good agreement. Thus, the proposed model provides good reference for research on irradiation reliability of uniaxial strained Si Nano NMOSFET.

\section{Acknowledgements}

This work was supported by the National Natural Science Foundation of China (Grant No. 61474085) and the Science Research Plan in Shaanxi Province, China (Grant No. 2016GY-085). 